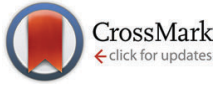

Cite this: Phys. Chem. Chem. Phys., 2016, 18, 22021

Received 15th July 2016

Accepted 18th July 2016

DOI: $10.1039 / c 6 c p 04935 d$

www.rsc.org/pccp

\section{Acetate ligands determine the crystal structure of CdSe nanoplatelets - a density functional theory study $\dagger$}

\author{
Rik S. Koster, ${ }^{* a}$ Changming Fang, ${ }^{\text {ab }}$ Alfons van Blaaderen, ${ }^{a}$ Marjolein Dijkstra ${ }^{a}$ and \\ Marijn A. van Huis ${ }^{a}$
}

\begin{abstract}
Cadmium selenide ( $\mathrm{CdSe}$ ) nanoplatelets of a few atomic layers thick exhibit extremely sharp photoluminescence peaks and are synthesized in the zinc blende crystal structure, whereas the most stable bulk polymorph of $\mathrm{CdSe}$ is the wurtzite structure. These platelets can be synthesized very monodispersely in thickness, and are covered with acetate ligands. Here, we show by means of density functional theory (DFT) calculations that these ligands play a pivoting role in the stabilization of 2D nanosheets as a whole, including the deviating crystal structure. The relative stability as a function of slab thickness, strong effects on electronic properties, and implications for synthesis are discussed.
\end{abstract}

\section{Introduction}

In the past decade, the interest in two dimensional (2D) nanomaterials has increased dramatically, since these show many desirable physical and electronic properties, often very different from their bulk counterparts. This revolution, which started with graphene, ${ }^{1}$ now includes a large variety of materials, such as hexagonal boron nitride (h-BN), ${ }^{2,3}$ metals, ${ }^{4,5}$ metal oxides, ${ }^{6,7}$ transition metal dichalcogenides (TMDs) ${ }^{2,8}$ and semiconductors. Thicknesses of such materials can range from a single atomic layer to tens of atomic layers, while the size in lateral dimensions is at least an order of magnitude larger. Such nanoplatelets, nanosheets or nanomembranes show great promise for applications in flexible electronics, biomedics, optoelectronics, and catalysis.

Whereas materials that have a bulk layered crystal structure, e.g. graphene, h-BN or TMD's, can be exfoliated ${ }^{9}$ to produce 2D nanoparticles, materials that do not have such a structure need to be synthesized by other methods. A frequently used method is using e.g. chemical vapor deposition to produce a thin layer

\footnotetext{
${ }^{a}$ Soft Condensed Matter, Debye Institute for Nanomaterials Science,

Utrecht University, Princetonplein 5, 3584 CC Utrecht, The Netherlands.

E-mail: r.s.koster@uu.nl,m.a.vanhuis@uu.nl

${ }^{b}$ Brunel Centre for Advanced Solidification Technology (BCAST),

Brunel University London, Uxbridge, Middlesex, UB8 3PH, UK

$\dagger$ Electronic supplementary information (ESI) available: Density of states plots for CdSe slab calculations. See DOI: 10.1039/c6cp04935d
}

on top of a sacrificial layer, which is subsequently removed leaving the nanosheets freely dispersed. However, this method requires sophisticated equipment, and the lateral dimensions of the resulting particles are difficult to control. A third route is to synthesize nanosheets bottom-up in solution, which allows production of these nanoparticles in larger quantities and achieving more control on their lateral dimensions. The results are highly dependent on the exact chemical details of the synthesis path followed, and currently syntheses are developed on a per case basis, as their underlying mechanisms are often not completely understood. The reader interested in the solution based synthesis methods is referred to the review paper by Wang and Wang. ${ }^{10}$

One particular system of interest is formed by zinc blende (ZB) cadmium selenide (CdSe) nanoplatelets as recently described by Ithurria and Dubertret. ${ }^{11}$ The thickness of these platelets is only several atomic layers and can be accurately controlled. The exposed (001) surface is atomically flat, while the lateral $\langle 100\rangle$ and $\langle 010\rangle$ directions can extend to roughly $100 \mathrm{~nm}$, depending on the reaction time. The platelets act as $2 \mathrm{D}$ quantum wells and show an extremely sharp photoluminescence peak due to quantum confinement, ${ }^{12}$ making them a suitable candidate material for optoelectronic applications.

Remarkably, the nanoplatelets are synthesized in the ZB structure, whereas wurtzite (WZ) is the most stable bulk CdSe polymorph. This is likely due to the ligands used in the synthesis, although it is excluded that lamellar assembly is the ruling growth mechanism in this case, since the reaction can take place at temperatures too high for lamellar assembly. The presumption that the ligands are of importance was supported by Mahler et al., who showed that replacing carboxylic acids by oleylamine induced a (partial) phase transition in ZB CdSe quantum dots, and a similar effect should be expected here. ${ }^{13}$

The ligands in these 2D nano materials have a profound influence on their overall stability and physical properties. Therefore, the system as a whole, including ligands, needs to be evaluated quantum mechanically in order to understand why deviating crystal structures, in this case zinc blende (ZB) rather 
than wurtzite (WZ), are found. To this end, we use density functional theory (DFT) to calculate the total energy, lattice relaxation, and effective charges of relevant configurations, comparing CdSe WZ-(0001), ZB-(001) and ZB-(111) terminations with and without ligand coverage.

\section{Computational methods}

For the DFT calculations, the Vienna $a b$ initio Simulations Package (VASP) ${ }^{14}$ was used, employing the projector augmented wave method $(\mathrm{PAW})^{15}$ and the optvdW-B88 functional ${ }^{16}$ to include non-local van der Waals interactions. The cutoff energy for the wavefunctions was set to $800 \mathrm{eV}(1100 \mathrm{eV}$ for the augmentation wavefunctions) and a $22 \times 22 \times 1 k$-point grid was used (Monkhorst-Pack for tetragonal cells, gamma centered for hexagonal cells). Slabs with thicknesses of several atomic layers were used, for each of the surfaces WZ-(0001), ZB-(001) and ZB-(111). Because of the periodic boundary conditions, the slabs extend to infinity in the lateral dimensions. Both the top and bottom side of the slabs were terminated by $\mathrm{Cd}$ atoms and a vacuum of at least $22 \AA$ was used. Calculations were done both on bare slabs and on (fully) acetate covered slabs. The structures were allowed to relax until the forces were smaller than $0.03 \mathrm{eV} \AA^{-1}$. Slab thicknesses range from 3 to $11 \mathrm{CdSe}$ layers. Since the slabs are $\mathrm{Cd}$ terminated, it implies that an $n$-layer slab consists of $n+1$ Cd layers and $n$ Se layers. For the covered slabs, an acetate molecule is placed on both surface Cd atoms, such that the composition of the cell becomes $\mathrm{Cd}_{n+1} \mathrm{Se}_{n}\left(\mathrm{CH}_{3} \mathrm{COO}\right)_{2}$. Fig. 1 shows the relaxed molecular models of the slabs with $n=3$.

To gain an understanding of the charge distribution of the system, the charge on the atoms, as described by Bader, ${ }^{17}$ was calculated using the code by the Henkelmann group. ${ }^{18-20}$ Because the GGA functional is known to underestimate the band gap, the hybrid HSE06 functional as described by Heyd, Scuseria and Ernzerhof was used to investigate the band structure of the most stable configuration. ${ }^{21}$ For this calculation, the cutoff energy was set to $300 \mathrm{eV}$ (500 for the augmentation wavefunctions), and a mixing parameter $\alpha=0.35$ was used, since this value reproduced the WZ bulk band gap at $0 \mathrm{~K}$ accurately.

\section{Results and discussion}

The results of the total energy calculations are listed in Table 1, where energy differences are expressed between ZB slabs and the WZ-(0001) slab, for both the bare and the acetate covered slabs: $\Delta E_{(i j k)}^{\mathrm{Bare} / \mathrm{Ac}}(n)=E_{\mathrm{ZB}(i j k)}^{\mathrm{Bare} / \mathrm{Ac}}(n)-E_{\mathrm{WZ}(0001)}^{\mathrm{Bare} / \mathrm{Ac}}(n)$. The most relevant case, $\Delta E_{(001)}$, is also shown in Fig. 2.

It is clear that for the uncovered slabs, the energy difference between the ZB-(001) slabs and the WZ-(0001) slabs is positive, i.e. the WZ-(0001) terminated slabs have a much lower energy than the ZB-(001) slabs. As can be expected from the similar crystal structures, the WZ-(0001) and ZB-(111) slabs are similar in energy.

By contrast, the acetate covered slabs have the lowest energy for slabs with the ZB-(001) surface, for all thicknesses calculated.
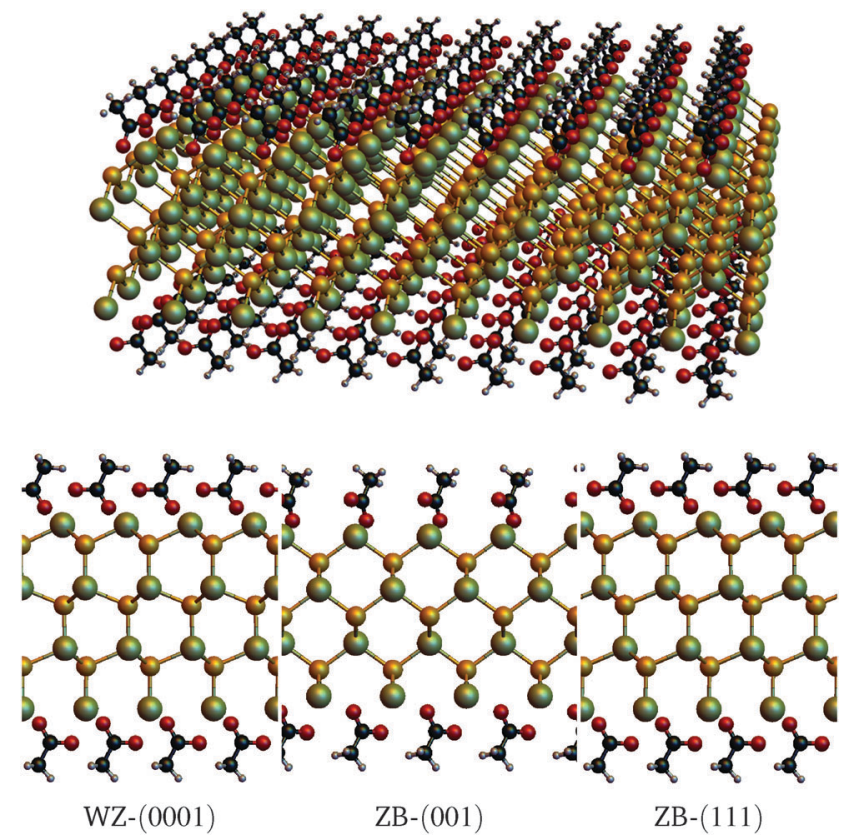

Fig. 1 Top: Perspective view of a CdSe ZB-(001) nanosheet covered with acetate ions $\left(\mathrm{Cd}_{4} \mathrm{Se}_{3}\left(\mathrm{CH}_{3} \mathrm{COO}\right)_{2}\right)$. Bottom: Side views of the acetate covered WZ-(0001), ZB-(001), and ZB-(111) $\left(\mathrm{Cd}_{4} \mathrm{Se}_{3}\left(\mathrm{CH}_{3} \mathrm{COO}\right)_{2}\right)$ slabs. For clarity, four unit cells have been plotted. Green: $\mathrm{Cd}$, yellow: Se, black: C, light grey: H, red: $O$.

Table 1 Energy differences between the ZB slabs and the WZ-(0001) surfaced $\mathrm{Cd}_{n+1} \mathrm{Se}_{n}$ slabs for different values of slab thickness $n$, e.g., $\Delta E_{(111)}^{\mathrm{Bare}}$ is the difference between the bare ZB-(111) and the bare WZ-(0001) slabs

\begin{tabular}{llcll}
\hline$n$ & $\Delta E_{(001)}^{\mathrm{Bare}}(\mathrm{eV})$ & $\Delta E_{(111)}^{\mathrm{Bare}}(\mathrm{eV})$ & $\Delta E_{(001)}^{\mathrm{Ac}}(\mathrm{eV})$ & $\Delta E_{(111)}^{\mathrm{Ac}}(\mathrm{eV})$ \\
\hline 3 & 0.71 & 0.02 & -1.06 & -0.03 \\
4 & 0.65 & 0.01 & -1.15 & -0.03 \\
5 & 0.61 & 0.00 & -1.20 & -0.03 \\
7 & 0.55 & -0.03 & -1.33 & -0.09 \\
9 & 0.53 & -0.04 & -1.36 & -0.09 \\
11 & 0.51 & -0.06 & -1.38 & -0.10
\end{tabular}

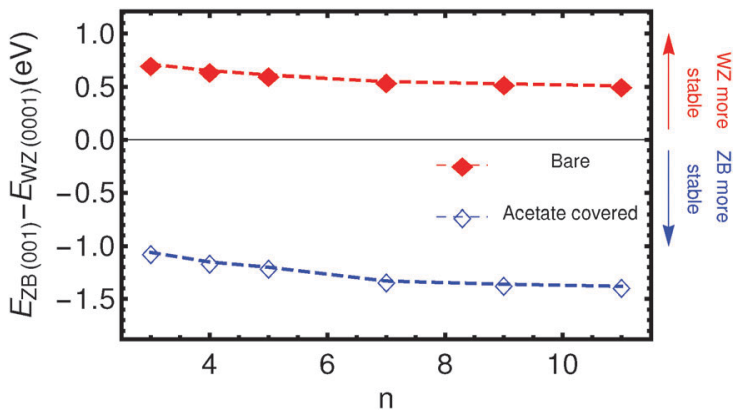

Fig. 2 The energy difference $\Delta E$ between the ZB-(001) and WZ-(0001) platelets, as a function of slab thickness, denoted with filled red squares for bare slabs $\left(\mathrm{Cd}_{n+1} \mathrm{Se}_{n}\right)$, and open blue squares for acetate covered slabs $\left(\mathrm{Cd}_{n+1} \mathrm{Se}_{n}\left(\mathrm{CH}_{3} \mathrm{COO}\right)_{2}\right)$.

This clearly shows that the stabilization of the ZB-(001) nanoplatelets can be attributed to the adsorption of the acetate ions. Fig. 2 clearly shows that this stabilising effect does not decrease with increasing thickness, at least for the thicknesses of the 


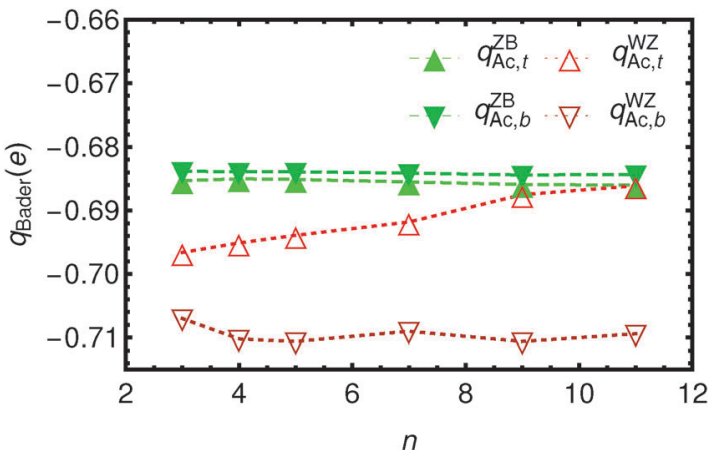

Fig. 3 The total Bader charge on the acetate ions as a function of the number of CdSe layers $n$. Triangles pointing up are used to denote acetate ions at the top of the $\mathrm{Cd}_{n+1} \mathrm{Se}_{n}$ slabs (open for WZ-(0001) slabs, closed for ZB-(001)), and solid triangles denote those at the bottom. Lines are drawn to guide the eye.

slabs calculated. Two aspects are of importance here; the number of ligands covering the slabs per unit area and the charge redistribution. First, the adsorption of neighbouring acetate ions might be energetically more unfavourable for WZ slabs than for ZB-(001) slabs, since the surface area per acetate ion is smaller for WZ slabs $\left(16.93 \AA^{2}\right)$ than for ZB slabs $\left(19.27 \AA^{2}\right)$. However, we stress that the number of acetate molecules per $\mathrm{Cd}_{n+1} \mathrm{Se}_{n}$ is the same, so that the energies of slabs with the same thickness can be directly compared. Second, the charge redistribution could be significantly different for the two surfaces. To quantify this, Bader charges were calculated.

In Fig. 3 and 4 the Bader charges on the acetate ions and the slabs, respectively, are shown. The charge transfer from the zinc blende(001) slab to the acetate ions is approximately $0.685 e$, for both the top and the bottom acetate ion, due to symmetry, and irrespective of the slab thickness. It is clear that the WZ(0001) slabs show a larger charge on the acetate ions. Moreover, since there is no mirror symmetry in the $x y$-plane, the charge on the bottom acetate differs somewhat from the charge on the top slab.

For a more qualitative description of the charge redistribution, isosurfaces of the electron density difference $\delta \rho$ are plotted in Fig. 5. Here, $\delta \rho$ is defined as

$$
\delta \rho(\vec{r})=\rho_{\text {slab }+2 \mathrm{Ac}}(\vec{r})-\rho_{\text {slab }}(\vec{r})-\rho_{\text {Ac,top }}(\vec{r})-\rho_{\text {Ac,bottom }}(\vec{r}),
$$

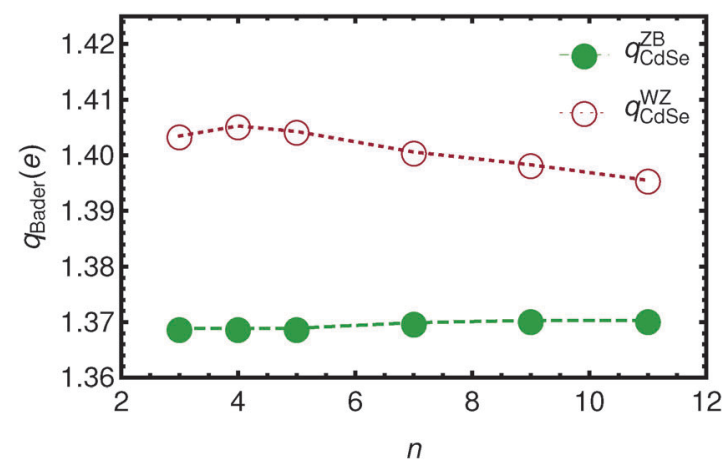

Fig. 4 The total Bader charge on the $\mathrm{Cd}_{n+1} \mathrm{Se}_{n}$ slabs a function of $n$. Open circles denote the WZ-(0001) slabs, while solid circles represent the ZB(001) slabs. Lines are drawn to guide the eye.
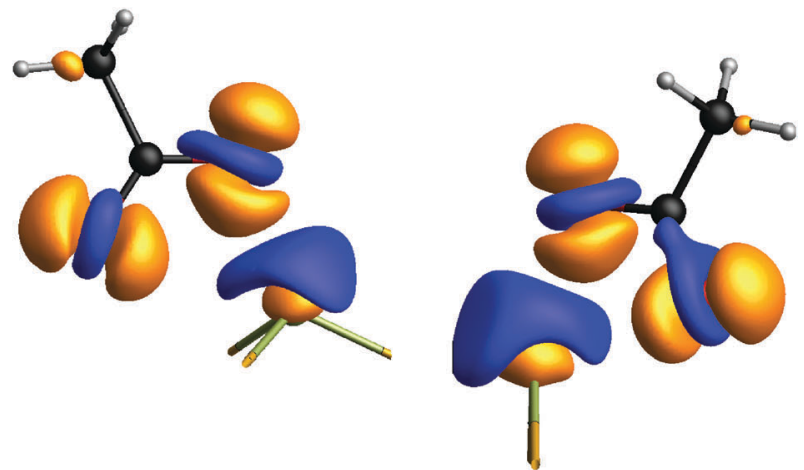

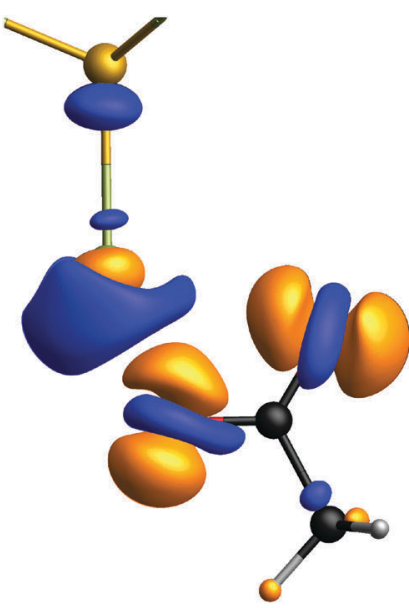

WZ-(0001)

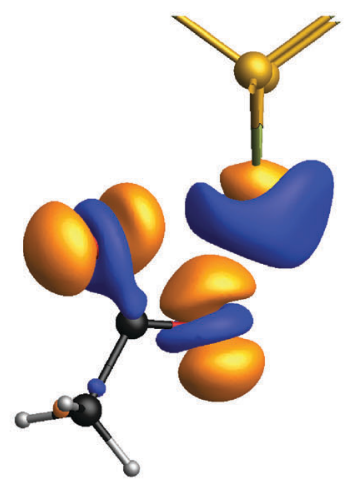

ZB- $(001)$
Fig. 5 Electron density differences $\delta \rho$ at the top and bottom of the slabs. Isosurfaces $\delta \rho=0.03 \AA^{-3}$ (orange) and $\delta \rho=-0.03 \AA^{-3}$ (blue) are shown.

where $\rho_{\text {slab }+2 \mathrm{Ac}}$ is the electron density of the complete system, $\rho_{\text {slab }}(\vec{r})$ is the electron density obtained for a static calculation of only the CdSe slab, and $\rho_{\mathrm{Ac}, \text { top/bottom }}(\vec{r})$ the electron densities of only the top/bottom acetate molecule. This effectively shows how the electrons are redistributed due to the acetate molecule covering the surface. Isosurfaces at $\delta \rho(\vec{r})= \pm 0.03$ are shown in Fig. 5. The oxygen atoms show a net gain in electron density, with an increase directly above the $\mathrm{O}$ atoms and a (smaller) decrease at the $\mathrm{O}$ position. Consequently, the $\mathrm{Cd}$ atom covered by the acetate shows a net decrease in electron density, with an increase directly at the Cd position and a (larger) decrease in the area above it. For the bottom WZ acetate, the charge redistribution seems to extend to the Se atom in the subsurface layer.

In the ESI $\dagger$ the projected densities of states (pdos) are shown for the bare and covered slabs. The off-stoichiometry has introduced states at the Fermi energy for both bare slabs and the WZ covered slab, making these slabs conducting instead of semi-conducting. Since the CdSe ZB-(001) slabs are the most relevant, the band structure was calculated using the hybrid HSE06 functional. Fig. 6 shows a bandgap of $2.35 \mathrm{eV}$ for the acetate covered slabs, which is in very good agreement with the observed photoluminescence peaks at $2.42 \mathrm{eV}^{22,23}$ The bare slab mid-gap states, which consist mostly of outer layer Cd s-orbitals and selenium p-orbitals, disappear in the covered slab; 


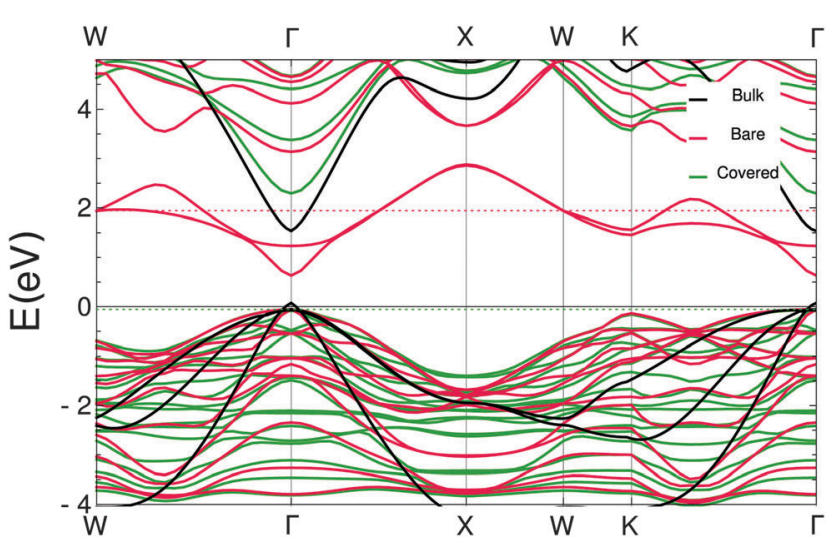

Fig. 6 Band structure of a ZB-(001) $\mathrm{Cd}_{5} \mathrm{Se}_{4}$ platelet, obtained with HSE. Bands of bulk, bare slabs, and covered slabs are plotted in one figure. For comparison the bands were shifted to match at the top of the valence band at the $\Gamma$ point. The dotted lines indicate the Fermi level.

the states in either the conduction or the valence band do not show such a strong contribution from the outer layer Cd atoms. The significant difference between the band structures of the bare and covered slabs show that it is essential to take the ligands into account when determining the electronic structure of this system.

We have shown by DFT calculations that the acetate ions play an essential role in stabilising the ZB structure for CdSe nanoplatelets. It should be noted however that all these DFT calculations are necessarily limited to the total energy calculations of small systems. They do not take into account the effect of the solvents or other molecules present, and neglect entropy effects as they are done at $0 \mathrm{~K}$. To explain the synthesis in full detail, the dynamics of the system must be understood. DFT calculations are not suitable for this and accurate MD simulations that would be required for that purpose are left for future work.

\section{Conclusion}

In summary, our DFT calculations show that indeed the absorption of stabilizing acetate ions onto CdSe nanoplatelets is responsible for the stabilization of the ZB crystal structure, even for nanoplatelets up to 11 layers thick. The ligands are crucial in determining the stability of this $2 \mathrm{D}$ nanomaterial as they induce a particular charge redistribution at the surface. The band gap for the most stable nanoplatelet that we calculated using the hybrid HSE06 functional is in excellent agreement with the energy of the experimentally observed photoluminescence. Our findings have considerable implications for a wide range of other 2D nanomaterials that are growth controlled by ligand manipulation, in particular those that are synthesized in an unusual crystal structure. ${ }^{24}$

\section{Acknowledgements}

M. A. v. H. acknowledges the Dutch science foundation NWO for a VIDI grant (grant number 723.012.006).

\section{References}

1 K. S. Novoselov, A. K. Geim, S. V. Morozov, D. Jiang, Y. Zhang, S. V. Dubonos, I. V. Grigorieva and A. A. Firsov, Science, 2004, 306, 666-669.

2 K. S. Novoselov, D. Jiang, F. Schedin, T. J. Booth, V. V. Khotkevich, S. V. Morozov and A. K. Geim, Proc. Natl. Acad. Sci. U. S. A., 2005, 102, 10451-10453.

3 A. Pakdel, Y. Bando and D. Golberg, Chem. Soc. Rev., 2014, 43, 934-959.

4 X. Huang, S. Tang, X. Mu, Y. Dai, G. Chen, Z. Zhou, F. Ruan, Z. Yang and N. Zheng, Nat. Nanotechnol., 2011, 6, 28-32.

5 J. Niu, D. Wang, H. Qin, X. Xiong, P. Tan, Y. Li, R. Liu, X. Lu, J. Wu, T. Zhang, W. Ni and J. Jin, Nat. Commun., 2014, 5, 3313.

6 Z. Sun, T. Liao, Y. Dou, S. M. Hwang, M.-S. Park, L. Jiang, J. H. Kim and S. X. Dou, Nat. Commun., 2014, 5, 3813.

7 T. Sasaki, M. Watanabe, H. Hashizume, H. Yamada and H. Nakazawa, J. Am. Chem. Soc., 1996, 118, 8329-8335.

8 V. Sorkin, H. Pan, H. Shi, S. Y. Quek and Y. W. Zhang, Crit. Rev. Solid State Mater. Sci., 2014, 39, 319-367.

9 V. Nicolosi, M. Chhowalla, M. G. Kanatzidis, M. S. Strano and J. N. Coleman, Science, 2013, 340, 1420.

10 F. Wang and X. Wang, Nanoscale, 2014, 6, 6398-6414.

11 S. Ithurria and B. Dubertret, J. Am. Chem. Soc., 2008, 130, 16504-16505.

12 S. Ithurria, M. D. Tessier, B. Mahler, R. P. S. M. Lobo, B. Dubertret and A. L. Efros, Nat. Mater., 2011, 10, 936-941.

13 B. Mahler, N. Lequeux and B. Dubertret, J. Am. Chem. Soc., 2010, 132, 953-959.

14 G. Kresse and J. Furthmüller, Comput. Mater. Sci., 1996, 6, 15-50.

15 P. E. Blöchl, Phys. Rev. B: Condens. Matter Mater. Phys., 1994, 50, 17953-17979.

16 J. c. v. Klimeš, D. R. Bowler and A. Michaelides, J. Phys.: Condens. Matter, 2010, 22, 022201.

17 R. F. W. Bader, Atoms in Molecules - A Quantum Theory, Oxford University Press, Oxford, 1990.

18 G. Henkelman, A. Arnaldsson and H. Jónsson, Comput. Mater. Sci., 2006, 36, 354-360.

19 E. Sanville, S. D. Kenny, R. Smith and G. Henkelman, J. Comput. Chem., 2007, 28, 899-908.

20 W. Tang, E. Sanville and G. Henkelman, J. Phys.: Condens. Matter, 2009, 21, 084204.

21 J. Heyd, G. E. Scuseria and M. Ernzerhof, J. Chem. Phys., 2003, 118, 8207-8215.

22 M. Olutas, B. Guzelturk, Y. Kelestemur, A. Yeltik, S. Delikanli and H. V. Demir, ACS Nano, 2015, 9, 5041-5050.

23 L. Biadala, F. Liu, M. D. Tessier, D. R. Yakovlev, B. Dubertret and M. Bayer, Nano Lett., 2014, 14, 1134-1139.

24 W. van der Stam, Q. A. Akkerman, X. Ke, M. A. van Huis, S. Bals and C. de Mello Donega, Chem. Mater., 2015, 27, 283-291. 